\title{
Chest compression during sustained inflation: hypoxic and oxidative stress
}

\author{
Shaun Cowan ${ }^{1,2 *}$, Georg M Schmölzer ${ }^{1,3}$, Tze-Fun Lee ${ }^{3}$ and Po-Yin Cheung ${ }^{1-4}$ \\ ${ }^{1}$ Centre for the Studies of Asphyxia and Resuscitation, Neonatal Research Unit, Royal Alexandra Hospital, Edmonton, Canada \\ ${ }^{2}$ Department of Pharmacology, University of Alberta, Edmonton, Canada \\ ${ }^{3}$ Department of Pediatrics, University of Alberta, Edmonton, Canada \\ ${ }^{4}$ Department of Surgery, University of Alberta, Edmonton, Canada
}

\begin{abstract}
Current recommendation for chest compressions in neonatal resuscitation is to use a coordinated 3:1 ratio of chest compressions to ventilations (C: $\mathrm{V}$ ), interrupting every third compression to deliver an assisted ventilation. Despite this recommendation, the evidence is still unclear on the optimal C:V ratio for use in neonates. Our team has previously reported a novel technique of sustained inflations with chest compressions (SI+CC) reduces time to return of spontaneous circulation and improves hemodynamic recovery. The objective of this study was to determine differences in hypoxic injury and oxidative stress markers of heart and lung in piglets resuscitated with $\mathrm{SI}+\mathrm{CC}$ compared to a coordinated 3:1 C:V. We found that the $\mathrm{SI}+\mathrm{CC}$ technique reduces oxidative stress in lung tissues and cumulative oxygen exposure with no differences in hypoxic injury. Our findings lend further support to transitioning this technique to neonatal resuscitation practice.
\end{abstract}

\section{Introduction}

Most newborn infants successfully transition from fetal to neonatal life [1]. However, approximately $10 \%$ of near-term and term deliveries will require some form of respiratory support during fetal to neonatal transition, whereas infrequently some newborns ( $0.08 \%)$ will require aggressive interventions such as chest compressions (CC) or epinephrine administration [1-3]. Neonatal resuscitation guidelines recommend CC if the heart rate remains $<60 /$ min despite adequate positive pressure ventilation [1]. Further, the guidelines recommend using a coordinated Compression: Ventilation $(\mathrm{C}: \mathrm{V})$ ratio of $3: 1$ if $\mathrm{CC}$ are needed. This approach is composed of $90 \mathrm{CC}$ and 30 inflations per minute, with a pause after every $3^{\text {rd }} \mathrm{CC}$ to deliver one effective ventilation. However the most effective $\mathrm{C}: \mathrm{V}$ ratio in newborns remains undetermined.

Neonatal heart rate $<60 / \mathrm{min}$ implies the inability of the heart to contract strongly enough to pump blood through the body and adequate oxygen delivery [3]. The aim of CC is to mechanically pump oxygenated blood through the body including the hypoxic myocardium until the myocardium becomes sufficiently oxygenated and recover with spontaneous function [4]. Achieving the return of spontaneous myocardial function as quickly as possible minimizes cellular and organ damage and is defined as return of spontaneous circulation (ROSC). Hypoxia and asphyxia depress myocardial function and promote maximal vasodilation with very low diastolic blood pressures leading to severe cellular damage. However, reperfusion and reoxygenation also cause significant cellular damage, and generate oxidative stress $[4,5]$. Dannevig et al. recently reported tissue genes levels of lung inflammation in severely asphyxiated newborn piglets resuscitated either $21 \%$ or $100 \%$ oxygen [6].

The current neonatal resuscitation guidelines recommend the use of $100 \%$ oxygen if CC are needed in newborn infants [1]. The resuscitation with $100 \%$ oxygen is associated with increased oxidative stress (e.g. increased lipid peroxidation in the brain and lungs [5,7]), increased mortality, and worsened neurological outcomes [7-9]. We recently demonstrated that superimposing $\mathrm{CC}$ with sustained inflation (CC+SI) compared to $3: 1 \mathrm{C}: \mathrm{V}$ in a piglet model of neonatal asphyxia significantly improve ROSC, mortality, as well as hemodynamic and respiratory parameters $[10,11]$. Although we observed significantly improved short-term outcomes using $\mathrm{CC}+\mathrm{SI}$, the effect on cellular or hypoxic damage and oxidative stress remains unanswered. The objective of the current study was to determine differences in hypoxic injury and oxidative stress markers of heart and lung in piglets resuscitated with either $\mathrm{CC}+\mathrm{SI}$ compared to 3:1 C:V. We hypothesized that hypoxic injury and oxidative stress markers would be lower in the $\mathrm{CC}+\mathrm{SI}$ group compared to the 3:1 C:V group.

\section{Materials and methods}

This study reports secondary outcomes of a randomized controlled biomedical trial that compared two approaches of CC using a swine model of neonatal asphyxia [10]. A total of 20 mixed breed piglets (age 1-4 days) with weight ranges of 1.6-2.1 kg were used in the original study. The piglets were obtained on the day of experimentation and the preparation began after acclimatization to the laboratory environment with no housing and husbandry requirements within our laboratory. These experiments were conducted in adherence to the guidelines of

Correspondence to: Georg M Schmölzer, M.D., Ph.D., Centre for the Studies of Asphyxia and Resuscitation, Neonatal Research Unit, Royal Alexandra Hospital, 10240 Kingsway Avenue NW, T5H 3V9, Edmonton, Alberta, Canada, Tel: +1 780735 4660; Fax: +1 780735 4072, E-mail: georg.schmoelzer@me.com

Key words: sustained inflation, chest compression, CPR, hypoxia, oxidative stress

Received: January 20, 2016; Accepted: February 17, 2016; Published: February 21, 2016 
Canadian Council of Animal Use and the University of Alberta Animal Use and Care Committee approved the study protocols. Reporting of results is and has been in accordance with the ARRIVE guideline for animal research reporting [12].

\section{CPR study protocol}

Our animal preparation protocol has been previously reported [13]. Briefly, as stated in our previous report [10] the preparation consisted of anesthesia with approximately 60-90 minutes of surgical instrumentation. A 60-minute recovery period followed instrumentation to allow physiological stabilization of the animals. Treatment groups were randomized to three groups: sham-operated $(n=4)$, 3:1 C:V group $(n=8)$, or CC-SI group $(n=8)$. Piglets were randomized to receive either coordinated CPR with 3:1 ratio or CC+SI. All piglets were exposed to 45-minute normocapnic hypoxia. Hypoxia was followed by an asphyxia period until heart rate decreased to $25 \%$ of baseline, which was achieved by disconnecting the ventilator and clamping the endotracheal tube. 15 seconds after heart rate reached $25 \%$ of baseline positive pressure ventilation was commenced for 30 seconds with a Neopuff T-Piece (Fisher \& Paykel, Auckland, New Zealand). The default settings were a peak inflating pressure of $30 \mathrm{~cm}$ $\mathrm{H}_{2} \mathrm{O}$, a positive end expiratory pressure of $5 \mathrm{~cm} \mathrm{H}_{2} \mathrm{O}$ and a gas flow of 8 $\mathrm{L} / \mathrm{min}$. CCs were performed using the two-thumb encircling technique by a single operator (GMS) in all piglets. A Metronome was used to achieve the targeted CC rate. After 30 seconds of CC, 100\% oxygen was commenced. Epinephrine was administered if no increase in heart rate or ROSC was observed despite adequate ventilation and CC. At 1 minute after CC were commenced, epinephrine $(0.01 \mathrm{mg} / \mathrm{kg}$ per dose) was given intravenously and then every minute as needed to a maximum of 4 doses. ROSC was defined as an increase in heart rate $>150 /$ min for 15 seconds. After ROSC piglets were allowed to recovery for four hours before the piglets were euthanized with an intravenous overdose of phenobarbital (100 mg/kg). The sham-operated group was randomized to the same surgical protocol, stabilization and equivalent experimental periods with no hypoxia, asphyxia nor resuscitation. CPR in the 3:1 group was performed according to the current resuscitation guidelines with $90 \mathrm{CC}$ per minute and 30 inflations. Piglets randomized to the SI group received a SI with a peak inflating pressure of $30 \mathrm{~cm} \mathrm{H}_{2} \mathrm{O}$ for the duration of $30 \mathrm{sec}$. During the SI, chest compressions with a rate of 120 per minute were provided. SI was interrupted after $30 \mathrm{sec}$ for one second before a further $30 \mathrm{sec}$ SI was provided. CC were delivered continuously until ROSC was achieved [10]. Tissue samples (including left ventricle and right lung) were taken at the end of experimentation and snap frozen in liquid nitrogen and stored at $-80^{\circ} \mathrm{C}$ until required for biochemical analysis.

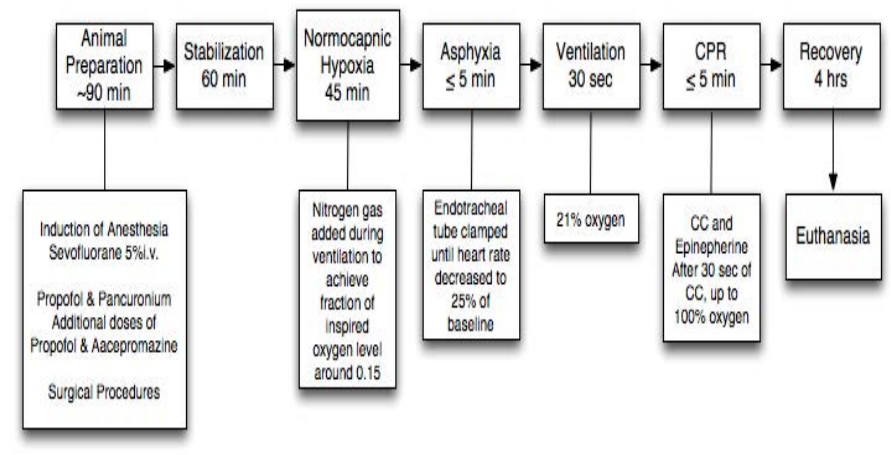

Figure 1. Study protocol flowchart, sham-operated piglets were instrumented, but did not receive hypoxic or reoxygenation treatment.

\section{Sample size}

The sample size for the nested biochemical analysis reported here was limited by the outcomes for our previous CPR study. Only those piglets surviving to the end of the 4-hour recovery period were suitable for comparison in this study. The resulting sample sizes were: shamoperated $(n=4), 3: 1 \mathrm{C}: \mathrm{V}$ group $(\mathrm{n}=3)$, and CC+SI group $(\mathrm{n}=7)$.

\section{Biochemical assays}

Determination of lactate and glutathione were used as surrogate markers for hypoxic damage and oxidative stress, respectively. Protein was determined to calculate total value per $\mathrm{mg}$ of protein to equate the individual results. Lactate was measured using an enzyme assay for absorbance of NADH at $340 \mathrm{~nm}$ (Sigma-Aldrich Canada Ltd., Oakville, $\mathrm{ON})$. Tissues for lactate assay were prepared by homogenization in a $6 \%$ perchloric acid with $0.5 \mathrm{mM}$ EGTA and then neutralized after separation with $5 \mathrm{M}$ potassium carbonate. Both total glutathione (GSH) and oxidized glutathione (GSSG) were measured using a commercially available ELISA kit (\#703002, Cayman Chemical, Ann Arbor, MI). Tissues were homogenized in a $0.1 \mathrm{M}$ phosphate buffer solution ( $\mathrm{pH}$ 7.4). GSH and GSSG were then assayed according to the assay kit instructions, with GSSG assayed by derivatizing GSH in the sample preparations with 2-vinylpyridine solution (Cat. \#13,229-2, Aldrich Chemical Company, Milwaukee, WI). Protein determination was measured using the bicinchoninic acid method using a bovine serum albumin standard and absorbance measured at $560 \mathrm{~nm}$ (SigmaAldrich Canada Ltd., Oakville, ON). All assay absorbance readings were measured using a Molecular Devices Spectramax 190 Absorbance Microplate Reader (Molecular Devices, LLC. Sunnyvale, California, United States).

\section{Alveolar oxygen exposure}

Alveolar oxygen exposure levels during the resuscitation were calculated by multiplying the partial pressure of alveolar oxygen by the time of exposure during resuscitation at $21 \%$ and $100 \%$ and summing the total exposure estimate. Alveolar oxygen partial pressure was estimated using the following equation:

$$
p_{a} O_{2}=F_{i} O_{2}\left(P_{A T M}-p H_{2} O\right)-\frac{p_{a} C O_{2}\left(1-F_{i} O_{2}(1-R Q)\right)}{R Q}
$$

Where $\mathrm{p}_{\mathrm{a}} \mathrm{O}_{2}$ is partial alveolar pressure of oxygen, $\mathrm{F}_{\mathrm{i}} \mathrm{O}_{2}$ is the fraction of inspired oxygen, $\mathrm{P}_{\text {ATM }}$ is the atmospheric pressure, $\mathrm{pH}_{2} \mathrm{O}$ is the vapor pressure of water, $\mathrm{p}_{\mathrm{a}} \mathrm{CO}_{2}$ is the partial alveolar pressure of carbon dioxide and RQ is the respiratory exchange ratio $[14,15]$. We utilized atmospheric pressure measurements at the time of resuscitation as recorded by the respiratory function monitor NM3 (Respironics, Philips, Andover, MA). Where values were missing, the mean (SD) of measured pressures was used $701 \pm 1 \mathrm{mmHg}$. We constrained the value for partial alveolar pressure of carbon dioxide and RQ to $40 \mathrm{mmHg}$ and 0.8 respectively, based on accepted physiological generalizations $[15,16]$. The vapor pressure of water was constrained to $47 \mathrm{mmHg}$, an estimated value for vapor pressure at $37^{\circ} \mathrm{C}$ [15]. The partial alveolar pressure of oxygen was the calculated for $\mathrm{F}_{\mathrm{i}} \mathrm{O}_{2}$ of 0.21 and 1.0.

The $\mathrm{p}_{\mathrm{a}} \mathrm{O}_{2}$ for each $\mathrm{F}_{\mathrm{i}} \mathrm{O}_{2}$ was then multiplied by the resuscitation time for each piglet and the oxygen exposure values at each $\mathrm{F}_{\mathrm{i}} \mathrm{O}_{2}$ were summed to total value for oxygen exposure during resuscitation.

\section{Statistical analysis}


Statistical results are reported as mean (standard error of mean) and median (interquartile range - IQR) for parametric and nonparametric variables, respectively. ANOVA analysis was used to compare treatments groups if normality tests passed and rank-order analysis was to be used otherwise. Student $t$-test was used for analysis of the cumulative alveolar oxygen exposure between treatment groups. The Pearson Moment test was used to analyze the correlation between the individual assayed biomarkers. $P$-values are 2 -sided and $\mathrm{p}<0.05$ was considered statistically significant. Statistical analyses were performed with Sigma Stat (Sigma Stat, San Rafael, CA, USA).

\section{Results}

A total of $3 / 8$ in the $3: 1 \mathrm{C}: \mathrm{V}$ group versus $7 / 8$ in the $\mathrm{CC}+\mathrm{SI}$ group ( $\mathrm{p}=0.0192$ ), and $4 / 4$ of the sham-operated piglets survived to 4 -hour recovery. Time to ROSC was significantly decreased in the CC+SI group with 38 (23-44) seconds, compared to 143 (84-303) seconds in the $3: 1$ group $(\mathrm{p}=0.0008)$ [10].

\section{Tissue lactate assay}

There was no difference in lactate levels measured in the left ventricle among groups: $0.96(0.19), 0.89(0.16)$, and $0.85(0.11) \mu \mathrm{mol} /$ mg protein for sham-operated, 3:1 C:V, and CC+SI groups, respectively $(\mathrm{p}>0.05)$ (Figure 2). Similarly, there was no difference in tissue lactate levels in the lungs with $0.16(0.05), 0.24(0.06)$, and $0.19(0.02) \mu \mathrm{mol} / \mathrm{mg}$ protein for sham-operated, $3: 1 \mathrm{C}: \mathrm{V}$, and $\mathrm{CC}+\mathrm{SI}$ groups, respectively ( $>0.05)$ (Figure 2).

\section{Tissue glutathione assay}

The tissue myocardial glutathione content (GSH and GSSG) was similar among groups in the left ventricle (Table 1). The glutathione
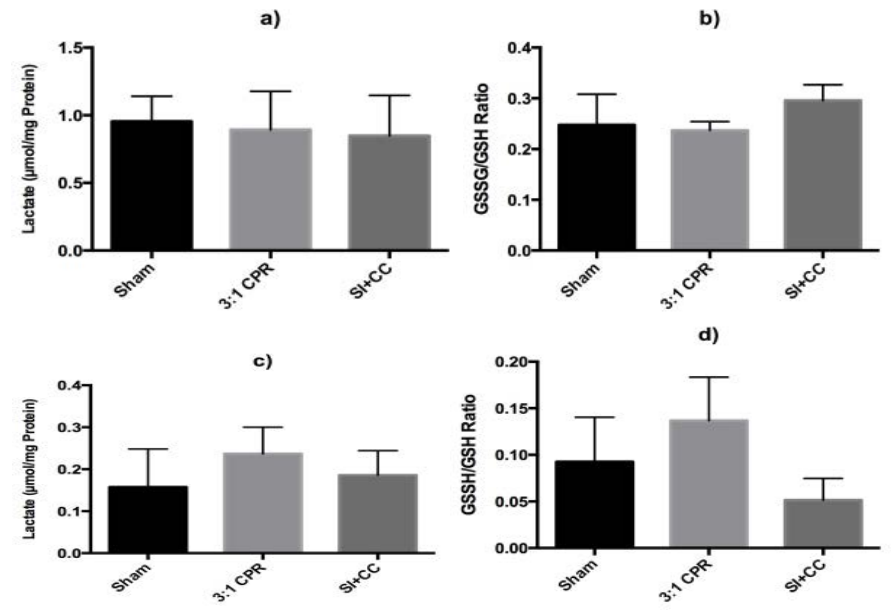

Figure 2. Tissue myocardial lactate (a) and GSSG/GSH ratio (b) in left ventricle, and tissue lactate (c) and GSSG/GSH (d) in the right lung. ${ }^{*} \mathrm{p}<0.05$ vs. 3:1 C:V group.

Table 1. Glutathione assay results for left ventricle and lung tissue samples, reported as group mean and (standard error) in CPR treatment type comparison. GSH: total glutathione, GSSG: oxidized glutathione

\begin{tabular}{|c|c|c|c|}
\hline \multirow{3}{*}{ Left Ventricle } & $\begin{array}{c}\text { GSH } \\
(\boldsymbol{\mu m o l} / \mathbf{m g} \text { protien }\end{array}$ & $\begin{array}{c}\text { GSSG } \\
(\boldsymbol{\mu m o l} / \mathbf{m g} \text { protien }\end{array}$ \\
\cline { 2 - 4 } & Sham-Operated & $351.63(25.89)$ & $88.23(13.45)$ \\
\cline { 2 - 4 } & $3: 1 \mathrm{C}:$ V Group & $344.52(65.61)$ & $87.57(14.05)$ \\
\cline { 2 - 4 } & SI+CC Group & $301.05(18.09)$ & $91.31(5.99)$ \\
\hline \multirow{2}{*}{$\begin{array}{c}\text { Right Lung, Lower } \\
\text { Lobe }\end{array}$} & Sham-Operated & $257.50(24.37)$ & $25.03(5.11)$ \\
\cline { 2 - 4 } & $3: 1 \mathrm{C}:$ V Group & $307.55(31.39)$ & $53.31(11.06)$ \\
\cline { 2 - 4 } & SI+CC Group & $233.39(21.15)$ & $12.67(2.13)$ \\
\hline
\end{tabular}

Table 2. Calculated Oxygen exposures for time exposed to $100 \%$ fraction inspired oxygen and total exposure based upon calculated aveolar partial pressures of oxygen

\begin{tabular}{|l|c|c|c|c|}
\hline & $\begin{array}{c}\text { Positive Pressure } \\
\text { Vent. At 21\% } \\
\text { (seconds) }\end{array}$ & $\begin{array}{c}\text { CPR Resuscitation } \\
\text { Time } \\
\text { (seconds) }\end{array}$ & $\begin{array}{c}\text { 100\% Oxygen } \\
\text { Exposure } \\
\text { (mmHg*s) }\end{array}$ & $\begin{array}{c}\text { Totla Exposure } \\
\text { Sum } \\
\text { (mmHg*s) }\end{array}$ \\
\hline $3: 1$ C:V Group & & & & \\
\hline$\# 1$ & 30 & 90 & 36840 & 53965 \\
\hline$\# 2$ & 30 & 77 & 28858 & 48564 \\
\hline$\# 3$ & 30 & 64 & 20876 & 40659 \\
\hline SI+CC Group & & & & \\
\hline$\# 1$ & 30 & 35 & 3075 & 26471 \\
\hline$\# 2$ & 30 & 45 & 9165 & 31293 \\
\hline$\# 3$ & 30 & 40 & 6120 & 29973 \\
\hline$\# 4$ & 30 & 43 & 7969 & 31531 \\
\hline$\# 5$ & 30 & 20 & 0 & 20357 \\
\hline$\# 6$ & 30 & 25 & 0 & 22616 \\
\hline$\# 7$ & 30 & 45 & 3070 & 32041 \\
\hline
\end{tabular}

(GSSG/GSH) ratio in the left ventricle were 0.25 (0.03), 0.23 (0.01), and 0.29 (0.01) for sham-operated, 3:1 C:V, and CC+SI groups respectively ( $p>0.05)$ (Figure 2). Lung tissue glutathione content was not different among groups (Table 1) and glutathione ratios were $0.09(0.02), 0.14$ (0.03), and 0.05 (0.01) for sham-operated, 3:1 C:V, and CC+SI groups, respectively with a significantly lower GSH ratio in the $\mathrm{CC}+\mathrm{SI}$ group (vs. 3:1 C:V group, $\mathrm{p}=0.047$ ) (Figure 2).

\section{Alveolar oxygen exposure}

The alveolar oxygen exposure during resuscitation was calculated by using the total resuscitation time, which included the initial 30 seconds of positive pressure ventilation prior to CPR. The exposure time of $21 \%$ and $100 \%$ inspired oxygen was multiplied by the partial alveolar pressure of oxygen calculated based on the above-described formula. The calculated cumulative alveolar oxygen exposure results are presented in Table 2. The cumulative alveolar oxygen exposure during resuscitation in the $\mathrm{CC}+\mathrm{SI}$ group was significantly lower sompared to the 3:1 C:V group 27755 (4706) and 47729 (6692) $\mathrm{mmHg}$ 'seconds, respectively (Student $t$-test, $\mathrm{p}<0.001)$. We noted a modest but insignificant correlation between alveolar oxygen exposure and tissue total and oxidized glutathione content $(r=0.63$ and $r=0.58 ; p=0.052$ and $\mathrm{p}=0.079$, respectively) but not between glutathione ratio and tissue lactate, in the lungs of asphyxiated piglets.

\section{Discussion}

We previously reported that $\mathrm{CC}+\mathrm{SI}$ significantly reduces ROSC, improves hemodynamic, and minute ventilation $[10,11]$. In addition, we reported that oxygen delivery and epinephrine usage was significantly reduced. In the current study we examined if $\mathrm{CC}+\mathrm{SI}$ would reduce hypoxic and oxidative stress injury. Tissue GSSG/GSH ratio, a reliable marker of intracellular oxidative stress and redox status was significantly increased in the lung of 3:1 C:V group. We observed a significant reduction in GSSG/GSH ratio in the SI+CC group compared to the $3: 1 \mathrm{C}: \mathrm{V}$ group. In addition, the calculated cumulative alveolar oxygen exposure was significantly reduced in the CC+SI group. These findings indicated that $3: 1 \mathrm{C}: \mathrm{V}$ group had higher oxygen exposure and increased oxidative stress, which potentially prolongs pro-oxidant status. Although we cannot categorically exclude increased oxidative stress in CC+SI group, it was lower compared to $3: 1 \mathrm{C}: \mathrm{V}$ group. The positive correlation of oxygen exposure to glutathione ratio in the lungs is supportive of reducing oxidative stress through shortened resuscitation time, especially if supplemental oxygen is used during 
CC. In addition, tissue lactate levels, markers of hypoxic injury were similar among groups. Interestingly, we found no correlation between glutathione ratios and lactate levels. In the current study decreased resuscitation time correlated with reduced glutathione ratio in the lung, which supports resuscitation techniques that improve ROSC and reduce oxygen exposure during $\mathrm{CPR}$ have the potential to reduce oxidative stress. This is further supported by a study by Mendler et al. who investigated different ventilation strategies and found no significant effect of different respiratory support strategies during CPR on ROSC [17]. This suggests that a combination of effective ventilation and CC-technique is required to improve ROSC $[18,19]$. With the data regarding oxidative stress reduction and lowered alveolar oxygen exposure without effect on hypoxic injury, we believe that the $\mathrm{CC}+\mathrm{SI}$ technique has the potential to improve patient outcomes if translated to neonatal resuscitation.

Possible confounders in this study could be related to the 4-hour recovery period, potentially masking larger differences in oxidative or hypoxic stress. The 4-hour recovery period may also have allowed physiological mechanisms to normalize the glutathione and lactate levels that could have been significantly different immediately following ROSC. While limited by the primary study design to monitor hemodynamic recovery, an alternative method for sampling of tissues immediately following ROSC may lead to different conclusions. This experimental model is strong with regard to translation as swine is a good model of neonatal asphyxia with similarities in physiological, biochemical, anatomical and developmental aspects, especially of the cardio-pulmonary system, between newborn humans and piglets $[13,20]$. The gradual onset of asphyxia is a good representation of the progressive nature of fetal distress that often occurs in utero prior to delivery [19]. A limitation of the model is the completed fetal to neonatal transition, which differs to newborn babies in the delivery room. A further limitation is the small sample size, as we only had tissues for biochemical analysis from piglets that survived the 4-hour period.

\section{Conclusion}

The 2010 guidelines in neonatal resuscitation recommend the use of $21 \%$ oxygen partly to reduce oxidative stress. Our findings demonstrated that $\mathrm{CC}+\mathrm{SI}$ resuscitation reduced the elevated lung glutathione ratio of piglets resuscitated by 3:1 C:V. The novel resuscitation method may further reduce alveolar oxygen exposure and lung tissue oxidative stress in asphyxiated neonates, in addition to the faster return of spontaneous circulation and improved hemodynamics.

\section{Acknowledgement}

GMS is a recipient of a Banting Postdoctoral Fellowship, Canadian Institutes of Health Research and an Alberta Innovates - Health Solutions Clinical Fellowship.

The study was supported by an operating grant to PYC from the Canadian Institutes of Health Research (MOP130483).

\section{References}

1. Perlman JM, Wyllie J, Kattwinkel J, Wyckoff MH, Aziz K, et al. (2010) Neonatal Resuscitation: 2015 International Consensus on Cardiopulmonary Resuscitation and Emergency Cardiovascular Care Science With Treatment Recommendations. Circulation 132: S204-S241. [Crossref]

2. Wyckoff M, Perlman J (2000) Cardiopulmonary resuscitation in very low birth weight infants. Pediatrics 106: 618-620. [Crossref]

3. Kapadia V, Wyckoff MH (2012) Chest compressions for bradycardia or asystole in neonates. Clin Perinatol 39: 833-842. [Crossref]
4. Gill RS, Pelletier JS, LaBossiere J, Bigam DL, Cheung PY, et al. (2012) Therapeutic strategies to protect the immature newborn myocardium during resuscitation following asphyxia.(Report). Can J Physiol Pharmacol 90: 689-695. [Crossref]

5. Solberg R, Longini M, Proietti F, Vezzosi P, Saugstad OD, et al. (2012) Resuscitation with supplementary oxygen induces oxidative injury in the cerebral cortex. Free Radic Biol Med 53: 1061-1067. [Crossref]

6. Dannevig I, Solevåg AL, Saugstad OD, Nakstad B (2012) Lung Injury in Asphyxiated Newborn Pigs Resuscitated from Cardiac Arrest - The Impact of Supplementary Oxygen, Longer Ventilation Intervals and Chest Compressions at Different Compression-to-Ventilation Ratios. Open Respir Med J 6: 89-96. [Crossref]

7. Kondo M, Itoh S, Isobe K, Kondo M, Kunikata T, et al. (2000) Chemiluminescence because of the production of reactive oxygen species in the lungs of newborn piglets during resuscitation periods after asphyxiation load. Pediatr Res 47: 524-527. [Crossref]

8. Perlman JM, Wyllie J, Kattwinkel J, Atkins DL, Chameides L, et al., (2010) Neonatal Resuscitation 2010 International Consensus on Cardiopulmonary Resuscitation and Emergency Cardiovascular Care Science With Treatment Recommendations. Circulation 122: 8516-8538. [Crossref]

9. Klinger G, Beyene J, Shah P, Perlman M (2005) Do hyperoxaemia and hypocapnia add to the risk of brain injury after intrapartum asphyxia? Arch Dis Child Fetal Neonatal Ed 90: F49-52. [Crossref]

10. Schmölzer GM, O'Reilly M, Labossiere J, Lee TF, Cowan S, et al. (2013) Cardiopulmonary Resuscitation With Chest Compressions During Sustained Inflations A New Technique of Neonatal Resuscitation That Improves Recovery and Survival in a Neonatal Porcine Model. Circulation 128: 2495-2503. [Crossref]

11. Li ES, Cheung PY, O'Reilly M, Schmölzer GM (2015) Change in tidal volume during cardiopulmonary resuscitation in newborn piglets. Arch Dis Child Fetal Neonatal Ed 100: F530-533. [Crossref]

12. Cheung PY, Gill RS, Bigam DL (2011) A swine model of neonatal asphyxia. J Vis Exp 56. [Crossref]

13. Curran-Everett D (2006) A classic learning opportunity from Fenn, Rahn, and Otis (1946): the alveolar gas equation. Adv Physiol Educ 30: 58-62. [Crossref]

14. Cruickshank S, Hirschauer N (2004) The alveolar gas equation. Continuing Education in Anaesthesia. Critical Care \& Pain 4: 24-27.

15. Widmaier EP, Raff H, Strang KT (2011) Vander's human physiology: the mechanisms of body function. McGraw-Hill, New York.

16. Lee KT (1986) Swine as animal models in cardiovascular research. Biomed Res 3: 1481-1486.

17. Mendler MR, Weber C, Hassan MA, Huang L, Waitz M, et al. (2015) Effect of Different Respiratory Modes on Return of Spontaneous Circulation in a Newborn Piglet Model of Hypoxic Cardiac Arrest. Neonatology 109: 22-30. [Crossref]

18. Li ES, Cheung PY, O'Reilly M, LaBossiere J, Lee TF, et al. (2016) Exhaled CO2 Parameters as a Tool to Assess Ventilation-Perfusion Mismatching during Neonatal Resuscitation in a Swine Model of Neonatal Asphyxia. PLOS ONE 11: e0146524-11. [Crossref]

19. Solevåg AL, Cheung PY, Lie H, O'Reilly M, Aziz K, et al. (2015) Chest compressions in newborn animal models: A review. Resuscitation 96: 151-155. [Crossref]

20. Schmölzer GM, O'Reilly M, Labossiere J, Lee TF, Cowan S, et al., (2014) 3:1 compression to ventilation ratio versus continuous chest compression with asynchronous ventilation in a porcine model of neonatal resuscitation. Resuscitation 85: 270-275. [Crossref]

Copyright: (C2016 Cowan S. This is an open-access article distributed under the terms of the Creative Commons Attribution License, which permits unrestricted use, distribution, and reproduction in any medium, provided the original author and source are credited. 